\title{
Longitudinal deformation of coronary stent detected by transesophageal echocardiography
}

\author{
Karin Kato $^{1 *}$ and Kiyoko Sato ${ }^{2}$ \\ ${ }^{1}$ Department of Anesthesia, Kyoto University Hospital, Sakyo-ku Kyoto, Kyoto, Japan \\ ${ }^{2}$ Department of Anesthesia, Mitsubishi Kyoto Hospital, Nisigyo-ku Kyoto, Kyoto, Japan
}

Received: October 18, 2016; Accepted: November 18, 2016, Published: November 23, 2016

*Corresponding author: Karin Kato, Department of Anesthesia, Graduate school of Medicine and Faculty of Medicine Kyoto University, Kyoto, Japan, 606-8507, Tel +81-075-751-3433; Fax+81-075-752-3259; E-mail: karin@kuhp.kyoto-u.ac.jp

\begin{abstract}
We describe a case of longitudinal stent deformation (LSD) detected by transesophageal echocardiography (TEE).LSD is a potentially serious complication, given that it has shown to be associated with stent thrombosis; therefore, anesthesiologists should be aware of a patient's LSD status prior to surgery so that they can take appropriate measures. Understanding how TEE can be used to detect LSD will, therefore, greatly benefit anesthesiologists. We report a case of LSD detection by TEE and describe the subsequent change in surgical procedure.
\end{abstract}

Keywords: Transesophageal echocardiography; Deformation; Stent

\section{Introduction}

More deliverable and flexible methods have enabled more complex coronary disease cases to be managed with percutaneous coronary intervention (PCI).Both strut thickness and the number of fixed connectors within cells has been reduced in new stents to help achieve increased flexibility. However, this has negatively affected other mechanical properties of the stents, including the resistance to longitudinal stress. Newer stent models pose the risk of longitudinal stent deformation (LSD). LSD was recently described as a mechanical complication that manifested after the implantation of thin-strut coronary stents [1]. The reported incidence of LSD is $0.2-1.0 \%$ [2, 3, and 4]. Although rare, LSD can potentially lead to serious complications, including restenosis, stent thrombosis, aneurysm formation, myocardial infarction, and cardiac death [1]. Most cases that have recently been reported, had utilized newer stent models; in these cases, LSD usually developed after guide catheter manipulation near ostially implanted stents or during re-crossing of freshly implanted stents [2]. LSD is usually detected by either angiography or intravascular ultrasound.

Despite the potential for severe adverse events, most anesthesiologists are unable to accurately detect LSD. Furthermore, they do not know how LSD appears on transesophageal echocardiography (TEE). In this report, we detail LSD detection by TEE.
Recognizing the risk for LSD is important, and it requires anesthesiologist to be particularly careful with patients who have stents. LSD can also occur secondary to a variety of mechanisms [2]. LSD identification and treatment are important because adverse events (e.g., stent thrombosis), which sometimes requires emergency revascularization, may occur.LSD detection during TEE can assist prompt patient management, guide treatment decisions, and help avert potentially serious complications.

\section{Case Report}

An 80-year-old male was referred to our hospital for chest pain that had recently worsened; the patient had gone into cardiopulmonary arrest three months prior to admission because of an acute myocardial infarction. During resuscitation, emergency catheter intervention revealed $99 \%$ and $90 \%$ stenosis of the left coronary main trunk (LMT) and right coronary artery (RCA), respectively. A drug-eluting stent (XiencePrime $2.5 \times 3 \mathrm{~mm}$, Boston Scientific and Abbott Vascular, Santa Clara, California; USA) was placed in the LMT and a bare metal stent (Integrity $3.5 \times 2$ mm Medtronic, Minneapolis, Minnesota, USA) was placed in the RCA. The Xience Prime stent was implanted near the ostium.

Electrocardiography showed ST segment depression and coronary angiography revealed $90 \%$ intra-stant stenosis of the LMT. An emergency coronary artery bypass graft (CABG) was scheduled for unstable angina. Intraoperative TEE showed that the stent implanted in the LMT had elongated $6 \mathrm{~mm}$ to the ascending arch; kinking of the proximal edge of the stent was also observed (Figure 1A). Epiaotic ultrasound results supported this finding (Figure 1B). The surgical procedure was changed to the open aortic artery technique. During surgery, we found that the stent had protruded into the aorta and that the stent had fractured (Figure 2). The LMT orifice was severely narrowed due to thickening of the vessel wall intima. The deformed section of the stent was respected during surgery, and the CABG was completed successfully. Post-pump TEE revealed shortened LMT stent (Figure 3).

\section{Discussion}

This report describes a case of LSD detection by TEE; to the 

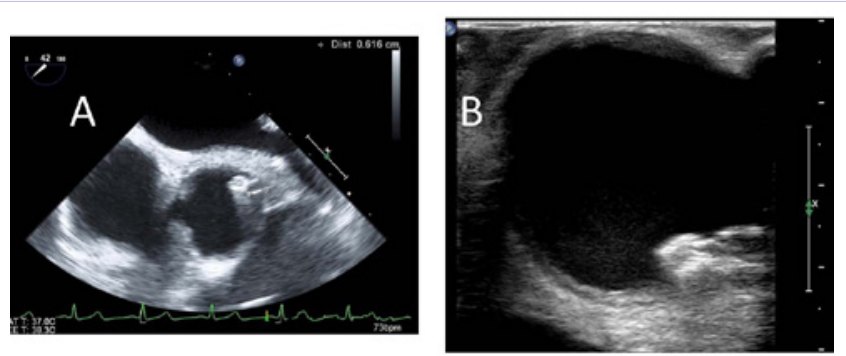

Figure 1: Intraoperative transoesophageal echocardiography $(\mathrm{A})$ and epidotic ultrasound (B).They both showed protrusion of the left main coronary trunk (LMT) stent toward the aorta.

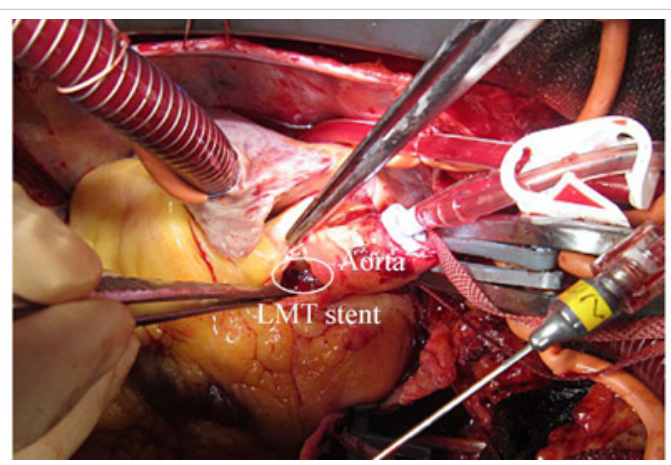

Figure 2: Intraoperative view during aortotomy. Open aortic surgery was performed to remove the proximal part of the fractured stent.

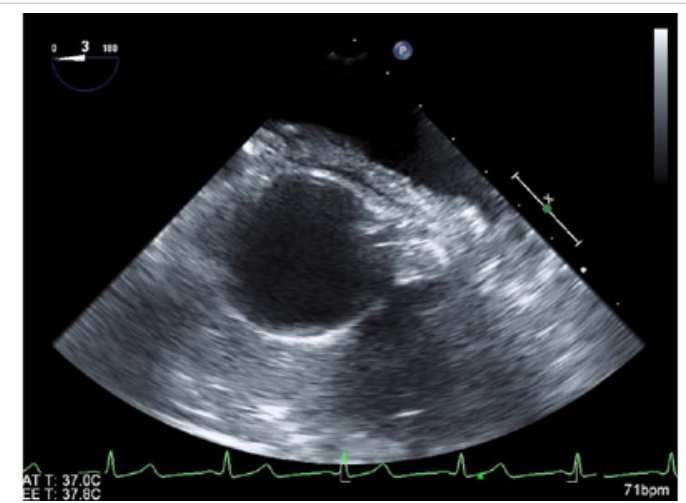

Figure 3: Intraoperative transoesophageal echocardiography after resection of fractured stent.

best of our knowledge, this has not been previously reported. There are, in fact, few published reports of clinical cases of LSD [5, 6].This unexpected intra-operative finding resulted in a change of surgical procedure.

In recent years, coronary stents have become thinner and, therefore, more deliverable. While these changes have enabled the treatment of more complex cases and lesions, particularly those formerly considered untreatable with PCI, they may affect longitudinal stent stability.

LSD poses challenges for PCI. Although LSD rarely occur and their impact on clinical outcomes is yet to be fully determined, they may lead to serious complications in some patients.LSD is the result of compression applied to the stent struts along the longitudinal axis by other devices, including guide catheters, post-dilatation balloons, or imaging tools. A particular subset of lesions, including ostial, bifurcation, and long lesions seem to be more vulnerable to LSD [7]. Almost half of all lesions associated with LSD are located in the left main coronary artery [2]. When treating left main lesions; stents are frequently undersized because the left main coronary artery is larger than currently available stents. As was noted in the present case, the most commonly observed LSD is near the proximal edge of the stent. This type of deformation may be caused by direct contact between the guiding catheter and the proximal edge stent struts that are not fully embedded into the vessel wall, resulting in stent malposition [3].

Treatment approaches for LSD are still being discussed. Some cases do not need revascularization and have no subsequent cardiac events [3]. Conversely, some cases require intervention. One mode of revascularization is post-dilatation of the stent or adding further stent in order to correct stent malposition. However, in some cases, including ours, this can be extremely hard because it is difficult for the guide catheter to pass through the distorted stent.

We are currently unsure of why transthoracic echocardiography (TTE) and angiography were unable to detect LSD in the present case. It may be hard to detect LSD by TTE as there are currently no reports that have reported LSD detection by TTE. Reduced stent visibility during radiography may result in LSD going undetected. LSD might either go undetected or be underestimated when using angiography alone. Alternatively, stent thrombosis might have already occurred, and the stent might have continued to fracture when the catheter for the angiography was pulled out. Another possibility is that LSD might have occurred when the patient suffered an acute myocardial infarction three months prior to admission. During resuscitation, there was a lot of activity at the site, so there was not enough time to expand the stent that subsequently deformed [2]. The LSD might have, therefore, accelerated in-stent thrombosis. We are unsure of the chronological sequence in which LSD and instent thrombosis occurred.

As the stent lumen was completely obstructed and disrupted, the deformation seemed to be irreversible. CABG was the only mode of revascularization possible, and the open aortic artery surgical technique was required to remove the deformed stent. Given that the follow-up angiography revealed obstruction by the distorted and protruding stent, resection of the stent was the only solution.

Successfully detecting LSD made a substantial difference to our clinical decision-making. Anesthesiologists should, therefore, be aware of LSD.TEE images can provide surgeons with accurate information prior to/during surgery. Additional careful perioperative assessment and monitoring are crucial. The increased numbers of reports on LSD have indicated the importance of carefully monitoring stents, especially when managing an ostial 


\section{lesion, as observed in this case.}

\section{References}

1. Mamas MA, Williams PD. Longitudinal stent deformation: Insights on mechanisms, treatments and outcomes from the food and drug administration manufacturer and user facility device experience database. EuroIntervention. 2012;8(2):196-204. doi: 10.4244/ EIJV8I2A33.

2. Inaba S, Weisz G, Kobayashi N, Saito S, Dohi T, Dong L, et al. Prevalence and anatomical features of acute longitudinal stent deformation: An intravascular ultrasound study. Catheterization and Cardiovascular Interventions. 2014;84(3):388-396. doi: 10.1002/ccd.25411.

3. Rigattieri S, Sciahbasi A, Loschiavo P. The clinical spectrum of longitudinal deformation of coronary stents: From a mere angiographic finding to a severe complication. J Invasive Cardiol. 2013;25(5):E101-105.
4. Abdel-Wahab M, Sulimov DS, Kassner G, Geist V, Toelg R, Richardt G. Longitudinal deformation of contemporary coronary stents: An integrated analysis of clinical experience and observations from the bench. J IntervCardiol. 2012;25(6):576-585. doi: 10.1111/j.15408183.2012.00765.x

5. Hanratty CG, Walsh SJ. Longitudinal compression: A "new" complication with modern coronary stent platforms--time to think beyond deliverability? EuroIntervention. 2011;7(7):872-877. doi: 10.4244/EIJV7I7A135

6. Williams PD, Mamas MA, Morgan KP, El-Omar M, Clarke B, Bainbridge A, et al. Longitudinal stent deformation: A retrospective analysis of frequency and mechanisms. EuroIntervention. 2012;8(2):267-274.

7. Shannon J, Latib A, Takagi K, Chieffo A, Figini F, Sacco F, et al. "Procedural trauma risks longitudinal shortening of the promus element stent platform”. Catheter CardiovascInterv. 2013;81(5):810- 